\title{
THE COHOMOLOGY OF AN ISOSPECTRAL FLOW ${ }^{1}$
}

\author{
DAVID FRIED
}

\begin{abstract}
Building on work of Tomei, we compute the cohomology of the manifold of real symmetric tridiagonal matrices with distinct fixed eigenvalues. The proof uses the global dynamical properties of the Toda flow on this isospectral manifold.
\end{abstract}

In this note we will extend some results of Tomei on the topology of the space $M$ of real symmetric tridiagonal $n \times n$ matrices with fixed eigenvalues $\lambda_{1}>\cdots>\lambda_{n}$ [T]. Tomei proved $M$ is a closed smooth manifold of dimension $d=n-1$, that $M$ is covered by $\mathbf{R}^{d}$, and he computed its Euler characteristic: we will compute the cohomology of $M$. For the corresponding results for the (homogeneous) space of all real symmetric $n \times n$ matrices with fixed spectrum see [B, BS, HPT].

We will make use of the Toda flow on $M$. For a matrix $L$ let $B(L)$ be the skew symmetric matrix that agrees with $L$ above the diagonal. The Lax equation $\dot{L}=[B(L), L]$ defines an isospectral flow on the space of $n \times n$ matrices. The Toda flow $\varphi_{t}$ is obtained by restricting to $M$. Tomei calculated $\chi(M)$ by applying the Poincaré-Hopf theorem to the Toda vector field. He showed that $\varphi_{t}$ is stationary at precisely the $n$ ! diagonal matrices $\operatorname{diag}\left(\lambda_{\pi(1)}, \ldots, \lambda_{\pi(n)}\right)=P(\pi)$, say, where $\pi$ is a permutation of $\{1, \ldots, n\}$. He also showed that $P(\pi)$ is hyperbolic with one unstable eigenvalue for each $j$ with $\pi(j)<\pi(j+1)$. This shows that the number of stationary points $P(\pi)$ with unstable dimension $u(\pi)=i$ is the Euler number $\left\langle\begin{array}{c}n \\ i+1\end{array}\right\rangle, i=0, \ldots, d$. We will show that this is also the $i$ th Betti number of $M^{2}$

Our results are based on the global behavior of $\varphi_{t}$. The unstable manifolds $W^{u}(\pi)$ of the $P(\pi)$ are Euclidean spaces: we will show that their closures $C^{u}(\pi)$ are closed $u(\pi)$-dimensional submanifolds of $M$ that can be arranged to form a spherical complex (see [G]). It follows that these $C^{u}(\pi)$ are an integral basis for $H_{*}(M ; \mathbf{Z})$. Indeed for any coefficient group $G, H_{i}(M ; G)$ and $H^{i}(M ; G)$ are the direct sum of $\left\langle\begin{array}{c}n \\ i+1\end{array}\right\rangle$ copies of $G$.

A dual complex is obtained by using $C^{s}(\pi)=$ the closure of the unstable manifold $W^{s}(\pi)$ of $P(\pi)$. The full cohomology ring of $M$ can be determined geometrically by intersecting a certain finite class of submanifolds that include the $C^{u}(\pi)$ and $C^{s}(\pi)$. One sees easily that $H^{*} M$ is generated by $H^{1} M$. We also obtain a presentation for $\pi_{1} M$ in which each relation is a commutator or a product of two commutators. Of course since $M$ is aspherical, all our cohomology computations give the group cohomology of $\pi_{1} M$.

\footnotetext{
Received by the editors August 28, 1985.

1980 Mathematics Subject Classification. Primary 57R19; Secondary 58F07, 58F09.

${ }^{1}$ This research was partially supported by the National Science Foundation and the Sloan Foundation.

${ }^{2} \mathrm{M}$. Davis has reproven this and generalized it in a context of reflection groups [D1, D2]. It is not now known how to get the cup product structure in that setting.
} 
We recall the known dynamics of $\varphi_{t}$. Let $a_{i}=L_{i i}$ be the diagonal coordinates on $M, i=1, \ldots, n$, and $b_{i}=L_{i, i+1}$ the off-diagonal ones, $i=1, \ldots, d$. The flow $\varphi_{t}$ is given by the equations $\left(b_{0}=b_{n}=0\right)$

$$
a_{i}^{\prime}=2\left(b_{i}^{2}-b_{i-1}^{2}\right), \quad b_{i}^{\prime}=b_{i}\left(a_{i+1}-a_{i}\right) .
$$

Observe that $\operatorname{sgn}\left(b_{i}\right)$ is constant on solutions and that $F(L)=\sum_{i=1}^{n}(n-i) a_{i}$ is strictly increasing along nonstationary solutions.

Taking all $b_{i}>0$, we can use Flaschka's coordinate change

$$
a_{k}=-\frac{1}{2} y_{k}, \quad b_{k}=\frac{1}{2} e^{\left(x_{k}-x_{k+1}\right) / 2}
$$

to convert the equations to the form $\left(x_{0}=-\infty, x_{n+1}=+\infty\right)$

$$
\ddot{x}_{k}=e^{x_{k-1}-x_{k}}-e^{x_{k}-x_{k+1}}, \quad k=1, \ldots, n .
$$

This is the finite Toda lattice of exponentially interacting mass points on the real line interacting under the Hamiltonian

$$
H=\frac{1}{2} \sum_{k=1}^{n} y_{k}^{2}+\sum_{k=1}^{n-1} e^{x_{k}-x_{k+1}}
$$

It is well known that the solution $\varphi_{t}$ converges as $t \rightarrow+\infty$ to

$$
P\left(\pi^{+}\right)=\operatorname{diag}\left(\lambda_{1}, \lambda_{2}, \ldots, \lambda_{n}\right)
$$

and as $t \rightarrow-\infty$ to

$$
P\left(\pi^{-}\right)=\operatorname{diag}\left(\lambda_{n}, \ldots, \lambda_{1}\right)
$$

(see $[\mathbf{M}]$ but note that the notation is different than that of $[\mathbf{T}]$, which we have followed).

Now consider an arbitrary $L \in M$. We will show

Proposition 1. There are $\pi_{L}^{+}, \pi_{L}^{-} \in S_{n}$ with $\varphi_{t} L \rightarrow P\left(\pi_{L}^{ \pm}\right)$as $t \rightarrow \pm \infty$.

To begin, let $Z(L) \subset\{1, \ldots, d\}$ be those $i$ for which $b_{i}=0$. Partition $\{1, \ldots, n\}$ into $|Z(L)|+1$ intervals $[\alpha, \beta]$ with $\beta \in Z(L) \cup\{n\}, \alpha-1 \in Z(L) \cup\{0\}$. Then $L$ decomposes as a direct sum $L=\bigoplus L_{\alpha, \beta}$ where each $L_{\alpha, \beta}$ is tridiagonal with nonzero subdiagonal entries. We order $\operatorname{Spec}\left(L_{\alpha, \beta}\right) \subset\left\{\lambda_{1}, \ldots, \lambda_{n}\right\}$ in an increasing way and define $\pi=\pi_{L}^{+} \in S_{n}$ by $\left\{\lambda_{\pi(\alpha)}, \ldots, \lambda_{\pi(\beta)}\right\}=\operatorname{Spec}\left(L_{\alpha, \beta}\right), \pi(\alpha)<\cdots<\pi(\beta)$. We define $\pi=\pi_{L}^{-} \in S_{n}$ by reversing the order in each interval: $\left\{\lambda_{\pi(\alpha)}, \ldots, \lambda_{\pi(\beta)}\right\}=$ $\operatorname{Spec}\left(L_{\alpha, \beta}\right), \pi(\beta)<\cdots<\pi(\alpha)$.

PrOOF OF PROPOSITION 1. Note that the equations decouple into $|Z(L)|+1$ independent systems of the same sort. This reduces to the case when all $b_{i} \neq 0$.

If all $b_{i}>0$, we are done. But there is an involution of the flow $\varphi_{t}$ that reverses the signs of a prescribed set of $b_{i}$ and leaves the $a_{i}$ and the other $b_{i}$ unchanged (these involutions were the key to Tomei's description of $M$ ). So one can always reduce to the case $b_{i}>0$. Since the involutions fix all the stationary points of $\varphi_{t}$, the result follows. Q.E.D.

We next describe the sets $C^{u}(\pi), \pi \in S_{n}$. Given $\pi$, we partition $\{1, \ldots, n\}$ into the maximal intervals $[\gamma, \delta]$ on which $\pi$ is decreasing, say $I_{1}, \ldots, I_{m^{-}}, m^{-}=m^{-}(\pi)$, and we denote this partition by $D^{-}(\pi)$. Also given $I \subset\{1, \ldots, n\}$ we write $M_{I}$ for the manifold $M$ associated to the $\lambda_{i}, i \in I$. 
Proposition 2. $C^{u}(\pi)=M_{I_{1}} \times \cdots \times M_{I_{m^{-}}} \subset M, I_{j} \in D^{-}(\pi)$.

Proof. Say $L \in W^{u}(\pi)$, so $\pi=\pi_{L}^{-}$. The partition determined by $Z(L)$ refines $D^{-}(\pi)$. Thus $W^{u}(\pi)$ lies in the indicated product, hence so does its closure.

The opposite inclusion follows by perturbing the summands $L_{\gamma, \delta},[\gamma, \delta]=I_{j}$, so that its subdiagonal entries are nonzero. This perturbed $L$ lies in $W^{u}(\pi)$. Q.E.D.

Clearly the flow $\varphi_{t}$ on $C^{u}(\pi)$ is the product of the Toda flows in each factor $M_{I_{j}}$; i.e., there is no coupling.

Of course the $C^{s}(\pi)$ have a corresponding description. Partition $\{1, \ldots, n\}$ into $J_{1}, \ldots, J_{m^{+}}, m^{+}=m^{+}(\pi)$, where $J_{i}$ are the maximal intervals on which $\pi$ is increasing. Then one has $C^{s}(\pi)=M_{J_{1}} \times \cdots \times M_{J_{m}+}$.

We now study the spherical cell complex on $M$. We defer the proof of the following technical fact. $B_{d}$ denotes the closed unit ball in $\mathbf{R}^{d}$.

PROPOSITION 3. There is a map $B_{d} \rightarrow M$ with int $B_{d} \cong W^{u}\left(\pi^{+}\right)$.

Let $X=M-W^{u}\left(\pi^{+}\right) \subset\left\{L \mid b_{1} \cdots b_{d}=0\right\}$. Then Proposition 3 says $M$ is obtained from $X$ by gluing on a $d$-cell. By $[\mathbf{T}] \operatorname{dim} X=d-1$ and $H_{d}(X ; \mathbf{Z})=\mathbf{Z}$, so this cell must be attached in a homologically trivial way, i.e., $\partial B_{d} \rightarrow X$ is zero on $H_{d-1}$. For each $\pi$ it follows that $C^{u}(\pi)=M_{I_{1}} \times \cdots \times M_{I_{m}-}$ is obtained from $X_{\pi}=C^{u}(\pi)-W^{u}(\pi)$ by attaching a top-dimensional cell in a homologically trivial way.

Now order $S_{n}$ linearly so that $\pi_{1}<\pi_{2} \Rightarrow F\left(P\left(\pi_{1}\right)\right) \geq F\left(P\left(\pi_{2}\right)\right)$. Then $\pi_{1}<\pi_{2} \Rightarrow W^{u}\left(\pi_{1}\right)$ is disjoint from $W^{s}\left(\pi_{2}\right)$. (Such orderings arise naturally in Smale's study of Axiom A systems [S]: one says that $\varphi_{t}$ is an Axiom A flow with $\Omega$ finite and no cycles.) Then $X_{\pi} \subset \bigcup_{\pi<\pi^{\prime}} C^{u}\left(\pi^{\prime}\right)$ for all $\pi$. It follows that $M$ is a spherical cell complex with open cells the unstable manifolds $W^{u}(\pi)$, and all attaching maps trivial on homology. Thus the homology and cohomology of $M$ are as stated previously.

It was critical for the above that $M$ be oriented: this can be seen differently by regarding $M$ as a level set of the characteristic polynomial map

$$
\left\{\begin{array}{c}
\text { symmetric tridiagonal } \\
n \times n \text { matrices }
\end{array}\right\} \rightarrow\left\{\begin{array}{c}
\text { monic polynomials of } \\
\text { degree } n
\end{array}\right\}
$$

and applying the implicit function theorem. Since both domain and range are oriented, any regular level set is oriented too. This even shows that $M$ is stably parallelizable and also that $M$ is independent of the choice of $\lambda_{i}$ 's.

We now consider the cases $d \leq 3$. For $d=1, \varphi_{t}$ is the north pole-south pole flow on $S^{1}$. For $d=2, M$ is a genus 2 surface divided by the curves $b_{1}=0, b_{2}=0$ into four hexagons with $\varphi_{t}$ as shown in Figure 1. Our spherical complex structure is also indicated: one should attach the $e_{i}$ in order, then the 2-cell. Note also the action of $\mathbf{Z}_{2}^{d}$ by reflections.

For $d=3, M / Z_{2}^{3}$ is an orbifold obtained from the truncated octahedron by mirroring all the faces with dihedral angles $90^{\circ}$. The boundary of this orbifold can be represented by a planar graph with $\varphi_{t}$ acting on the edges as shown in Figure 2. The highest point is the source and the exterior quadrilateral and the highest two hexagons lie in its repelling basin. The other eleven 2-cells are of the form $C^{u}(\pi) / \mathbf{Z}_{2}^{3}$ and correspond to our basis for $H_{2}(M ; Z) \cong H^{1}(M ; Z)$. Each 2-cell represents a surface embedded in $M$, quadrilaterals corresponding to tori (the product of two 


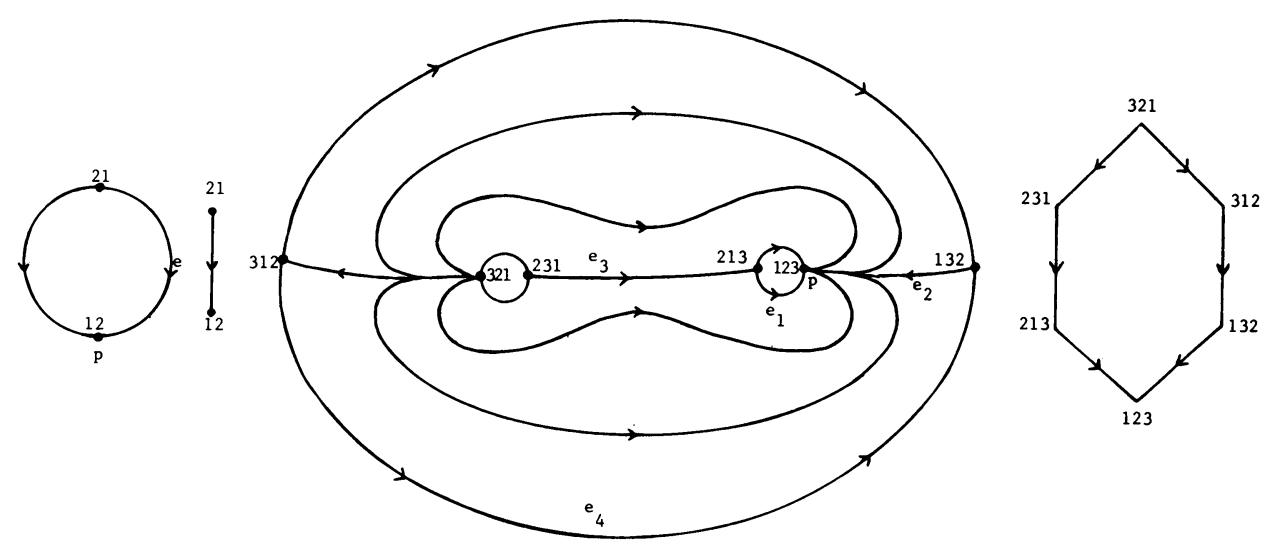

FIGURE 1. $d=2$
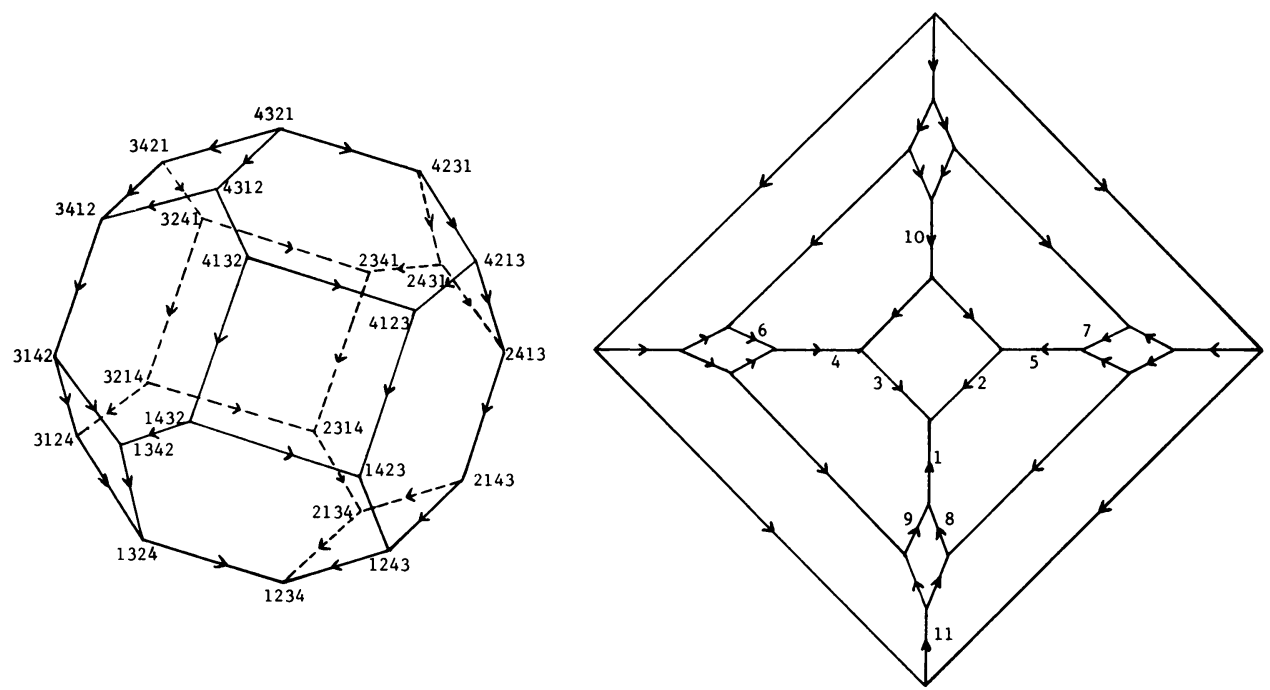

FIGURE 2. $d=3$

$d=1$ 's), hexagons to genus 2 surfaces $(d=2)$. These 14 surfaces satisfy three independent homologies arising from the regions $R_{i}=\left\{b_{i} \geq 0\right\}$ by setting $\partial R_{i}=0$. The quadrilaterals/tori are related using $R_{2}$; the hexagons/genus 2 surfaces use $R_{1}$, $R_{3}$. Each of these relations expresses one of the surfaces containing the source in terms of our basis.

Each edge in the graph represents an embedded circle in $M$. Each 2-cell contributes two relations, each asserting that the sum over alternate sides vanishes. A hexagon, for instance, corresponds to a genus 2 surface whose top half and front half give two homologies, each involving three circles. These relations enable edges near the source to be expressed in terms of those nearer the sink and eventually in terms of our basis of 11 generators.

Corresponding behavior holds for any $d$. Tomei produces a convex polytope in $\mathbf{R}^{d}$ homeomorphic to $M / \mathbf{Z}_{2}^{d}$. The $i$-cells in this polytope correspond to the $i$ - 
dimensional connected submanifolds $\Sigma_{i}$ of $M$ that arise as components of the sets defined by setting $d-i b_{j}$ 's equal to zero. Such components are determined by allocating eigenvalues to the various matrix blocks, and each is a product of lower dimensional $M$ 's (cf. the tori that arise for $d=3$ ).

The $(d-1)$-cells satisfy $d$ homologies arising from the boundaries of the sets where $b_{i} \geq 0$. These enable the $(d-1)$-manifolds passing through the source to be expressed in terms of the remaining ones, which are of the form $C^{u}(\pi)$, hence in our basis for $H_{d-1} M$. Applying such relations to the $\Sigma_{i}$ 's inside the $\Sigma_{i+1}$ 's expresses them in terms of the most downstream ones, the $C^{u}(\pi)$ in our basis.

With this procedure in mind, one sees how to compute the Poincaré duals of our basis: $C^{u}(\pi)$ is dual to $C^{s}(\pi)$, and this can be expressed in terms of $C^{u}\left(\pi^{\prime}\right)$ 's.

The cup product structure on $H^{*} M$ is also evident now. One forms the cup product of basis elements $\xi \in H^{i} M, \eta \in H^{j} M$ by intersecting the corresponding cells of dimension $d-i, d-j$ to obtain either a $d-i-j$ cell or some lower dimensional cell: the latter counts zero, and a $d-i-j$ cell is expressible in terms of our basis for $H^{i+j} M$. Of course, to determine the sign of the cup product, one must keep track of orientations.

Since every cell of dimension $<d$ is an intersection of $(d-1)$-cells (set the $b_{j}=0$ separately), we see that $H^{*} M$ is generated by 1 -dimensional classes. Indeed it has a presentation with one generator $\xi_{a}$ for each $(d-1)$-cell $a$, one linear relation in the $\xi_{a}$ obtained from setting $\partial R_{i}$ equal to zero for each $i=1, \ldots, d$, and one quadratic relation $\xi_{a} \cup \xi_{b}=0$ for each disjoint pair $a, b$. To see that these relations determine $H^{*} M$, we show that the algorithm of pushing cells downstream into our basis only uses such relations. But each $\Sigma_{i+1}$ can be written as the intersection of the $\Sigma_{d-1}$ 's containing it and by then intersecting with the linear relation $\partial R_{j}=0$ for each $j$ one gets all the linear relations amongst the $\Sigma_{i} \subset \Sigma_{i+1}$ used in the algorithm.

Finally we consider $\pi_{1} M=H$. In building our spherical complex, a 2-cell is either a torus, in which case the fundamental group acquires a relation that is a commutator, or a genus 2 surface, for which the relation is a product of two commutators. So $H$ has a presentation of greatest possible efficiency (fewest generators, fewest relations) in which all relations are commutators or products of two commutators.

The function $F=\sum(\dot{n}-i) a_{i}$ is Morse [T]. Our homology computation shows that it is a perfect Morse function, i.e., has as many critical points as the homology requires.

We summarize our results in

THEOREM. The unstable manifolds of stationary points for the Toda flow are the open cells of a spherical complex structure on $M$. Their closures are smooth manifolds. For any ring $R$, these form a free basis for $H^{*}(M ; R)$, with $n$ ! elements $\left\langle\begin{array}{c}n+1 \\ i\end{array}\right\rangle$ in each dimension $i$.

The manifolds obtained by setting certain $b_{i}=0$ and taking components correspond to the cells in Tomei's polytope $C$. They can be expressed in terms of our preferred basis by iterated use of relations arising from the bounded regions with certain $b_{i}=0$ and one $b_{i} \geq 0$. This determines the ring structure

$$
\left.H^{*}(M)=\left\langle\xi_{a} \in H^{1}(M)\right| \xi_{a} \cup \xi_{b}=0 \text { if } a, b \text { disjoint; } \partial R_{i}=0\right\rangle .
$$


The fundamental group $\pi_{1} M=H$ has a presentation with $\operatorname{dim} H^{1}(H)$ generators and $\operatorname{dim} H^{2}(H)$ relations.

We end by proving Proposition 3. Consider the regular cell complex for the universal cover $\tilde{M}$ of $M$ constructed by Tomei. The source of $\varphi_{t}$ lifts to a vertex $v \in \tilde{M}$ whose open star is the lift of the unstable manifold of the source. The closed star of $v$ is a ball of dimension $d$ obtained by reflecting the polytope around $v$ to all $2^{d}$ positions. Projecting back into $M$ proves the proposition.

\section{REFERENCES}

[B] A. Borel, Sur la cohomologie des espaces fibres principaux et des espaces homogenes de groupes de Lie compacts, Ann. of Math. (2) 57 (1953), 115-207.

[BS] R. Bott and H. Samelson, Applications of the theory of Morse to symmetric spaces, Amer. J. Math. 80 (1958), 964-1029.

[D1] M. Davis, Some aspherical manifolds, Ohio State Univ., preprint.

[D2] _ , The homology of a space on which a reflection group acts, Ohio State Univ., preprint.

[G] Marvin Greenburg, Lectures on algebraic topology, Benjamin, New York, 1967.

[HPT] W. Y. Hsiang, R. Palais and C. L. Terng, Geometry and topology of isoparametric submanifolds in Euclidean spaces, Proc. Nat. Acad. Sci. U.S.A. 82 (1985), 4863-4865.

[M] Jurgen Moser, Finitely many mass points on the line under the influence of an exponential potential-an integrable system, Lecture Notes in Phys., vol. 38, Springer-Verlag, Berlin and New York, 1975, pp. 467-497.

[\$] Steve Smale, The $\Omega$-stability theorem, Proc. Sympos. Pure Math., vol. 14, Amer. Math. Soc., Providence, R. I., 1968, pp. 289-298.

[T] Carlos Tomei, The topology of isospectral manifolds of tridiagonal matrices, Duke Math. J. 51 (1984), 981-996.

Department of Mathematics, Boston University, Boston, Massachusetts 02215 